\title{
Los padres de familia y la sexualidad en la adolescencia
}

\section{Parents and Sexuality in Adolescence \\ Maqtakunapa salinakuyninmanta yachana: tayta mamakunapa yachanan}

\author{
Gunther Balarezo López ${ }^{1}$ \\ Universidad Científica del Sur, Lima, Perú \\ gbalarezo52@gmail.com
}

José Manuel Balarezo Galarreta ${ }^{2}$

Universidad de Lima, Lima, Perú

smokky101@hotmail.com

\section{RESUMEN}

Se hizo una revisión bibliográfica de la literatura publicada sobre sexualidad en la adolescencia, y se analizaron y resumieron los aspectos más relevantes. La adolescencia es una etapa en que se hace frente a una serie de cambios biopsicosociales, en la cual el hogar debería ser el primer centro de aprendizaje de cuestiones relacionadas con la sexualidad; pero, en la realidad, esto no ocurre, debido a que los padres muchas veces no tienen los conocimientos adecuados. Como consecuencia, los adolescentes tienen mitos y tabúes al respecto y buscan respuestas a sus dudas en medios que no son los más apropiados.

\section{PALABRAS CLAVE}

Sexualidad, adolescencia, padres de familia

1 Sociólogo y magíster en Salud Pública, especialista en sociología de la salud, salud sexual y reproductiva, y metodología de la investigación científica. Ha sido becario de la Organización Mundial de la Salud (Brasil y Chile) y la Fundación Ford (Argentina), consultor del Fondo de Población de las Naciones Unidas en el Programa de Capacitación Laboral Juvenil (ProJoven, Ministerio de Trabajo) y docente de la Escuela Superior de Guerra Naval del Perú, la Universidad Peruana Cayetano Heredia y la Universidad Inca Garcilaso de la Vega. Ha participado en investigaciones sobre métodos anticonceptivos y aspectos sociales de la salud sexual y reproductiva. Autor de numerosos artículos en el Perú y el extranjero y del libro Parafilas: Glosario. Actualmente es docente en la Universidad Científica del Sur (Facultad de Medicina Humana), la Universidad Peruana de Ciencias Aplicadas (Escuela de Medicina) y en la Universidad Ricardo Palma (Maestría en Salud Pública).

2 Alumno del decimosegundo ciclo de la Facultad de Psicología, Universidad de Lima. 


\section{ABSTRACT}

It was a literature review of the published literature on adolescent sexuality, analyzing and summarizing the most relevant aspects. Adolescence is a stage that faces a number of changes bio-psycho-social, in which the home should be the first learning center issues related to sexuality, but in reality, this does not happen because that parents often do not have the right skills. As a result, teens have myths and taboos about and seek answers to their questions in ways that are best suited.

\section{KEYWORDS}

Sexuality, adolescence, parents

\section{PISILLAPI QILLQASQA}

Kay qillqasqaqa rimanqa, tukuy ima qillqasqakunata taqwirispa, maqtakunapa salinakuyninmanta. Chaykunata kuskispaqa yachasunchik imayna kasqanta, imata nisqankunatapas. Maqtakunaqa maqta kayninkupi tikrakunku tukuy kayninkuta. Paykunaqa hastawanmi tikrakamunku Iliw hatunyasqanrayku, wiñakusqanrayku. Kwirpunkuna, aychankuna, yuyariykunkunapas, paykuna kayninkunapas tikrakamunsi. Chaymi puntata wasipi tayta mamakunaqa yachachinman maqta churinkunata, paykuna salinakuyninmanta yachananpaq. Hinapas manan tayta mamakunaqa yachankuchu imatapas chay maqtakunapa salinakuyninmantaqa. Maqtakunataqmi manchakunku, tunkiykunku mana yachaspa maqtakayninkunamanta. Chaymi maskanku willaykunata huklawkunapi.

\section{TIQSI RIMANAKUNA}

Salinakuy, maqta kay, tayta mamakuna kay

La sexualidad es definida como:

Un conjunto de manifestaciones comportamentales, propias de la especie humana, influenciadas por factores biológicos, psicológicos y socioculturales, por medio de los cuales los individuos se manifiestan conforme a su identidad, sus roles y su orientación a lo largo de toda su vida (Portilla Valdivia y otros, 1997, p. 17).

La sexualidad es la manera en que una persona siente, piensa y actúa como ser sexual, dependiendo del género y del tipo de sociedad en que se encuentre; por lo tanto, la sexualidad no permanece estática, sino que 
es cambiante; es decir, no es la misma en todos los lugares y en todas las épocas. Su función se orienta hacia el desarrollo de la afectividad, comunicación y experiencias placenteras en toda relación humana (Portilla Valdivia y otros, 1997; Bendezú y otros, 1992; Salazar y otros, 1996; ASDE, 2002).

La sexualidad es un componente psicosocial importante en el desarrollo de todo ser humano, porque sienta las bases para que las personas se relacionen entre sí, debido a que se produce un intercambio de conocimientos, actitudes, prácticas, valores, etc., que en el futuro pueden influir en las relaciones con el sexo opuesto y/o la pareja. La sexualidad es aprendida desde que se nace y ese aprendizaje solo termina con la muerte (Bendezú y otros, 1992; ASDE, 2002).

Una de las etapas de la vida donde la tarea central es el logro de la identidad personal y sexual es la adolescencia, porque el joven se enfrenta con una «revolución fisiológica» por los profundos cambios físicos, psicológicos y sociales que amenazan su imagen corporal y su yo. Es una etapa donde comienza a preocuparse por lo que «parece ser» a los ojos de los demás, en comparación con el sentimiento que tiene de sí mismo (Rubarth, 1994; López Reyes y La Rosa Huertas, 1993).

Pero ¿qué significa la adolescencia? Etimológicamente, la palabra adolescencia proviene del latín adolescentia, y es una etapa del desarrollo entre la pubertad y la adultez (López Reyes y La Rosa Huertas, 1993). Según la Organización Mundial de la Salud (OMS) (Rubarth, 1994), la adolescencia es la etapa comprendida entre los 10 y 19 años, y se caracteriza por el acelerado crecimiento físico, al final del cual la persona alcanza precisamente la madurez física y sexual. En esta etapa el adolescente deja de ser niño, pero todavía no tiene la madurez ni el equilibrio emocional. Este es un periodo en el cual se produce un gran desarrollo físico, con un fuerte impacto en la vida psicológica y social. Dentro de los cambios sociales se tiene que el adolescente (Salazar y otros, 1996; Ministerio de Educación, 1998; Pick de Weiss y Vargas-Trujillo, 1992):

1. Establece una relación muy intensa con un grupo de pares, adoptando su lenguaje, vestimenta y costumbres, lo cual le permite afirmar su imagen y adquirir seguridad social.

2. Es capaz de hacer una revisión crítica de los valores aprendidos en la familia o en la escuela, lo cual es necesario para poder incorporarlos como propios.

3. Experimenta un gran sentido de justicia: aceptan un castigo si consideran que fue merecido, pero si fue algo injusto se le provoca una innecesaria rebeldía.

4. Adquiere responsabilidades de adulto, pero no hay una percepción de sus derechos. 
Además de los cambios sociales, los principales cambios sexuales producidos en los adolescentes son (ASDE, 2002):

1. Producto del funcionamiento hormonal, hay un incremento de sus impulsos sexuales, los cuales experimentará como bueno o malo según la educación y formación que haya recibido.

2. Las actitudes del adolescente hacia el sexo están influidas por la calidad de información recibida en esta materia; del mismo modo, por las actitudes de otras personas importantes como sus padres y sus experiencias personales.

3. Las actitudes de las personas que rodean al adolescente son diferentes, según el adolescente sea varón o mujer. Los padres generalmente son más represivos con las «hijas» que con los «hijos». Sin embargo, los varones reciben mayor presión social de parte de sus amigos para demostrar su virilidad.

En tal sentido, los adolescentes se ven afectados por problemas vinculados a sus estilos de vida y la sexualidad que se reflejan en la iniciación sexual temprana, embarazos no deseados, abortos y ejercicio de la sexualidad sin la debida protección, exponiéndose a contraer infecciones de transmisión sexual (ITS), entre ellas el VIH/SIDA (PROJoven, 1999).

En nuestro país, el inicio de las relaciones sexuales está asociado con el nivel de educación. El promedio es de 13,7 años; en las mujeres es de 14,4 años y de 13,5 años en los hombres. En los últimos 13 años, la tasa de madres adolescentes en el Perú se mantiene casi sin variación; del total de adolescentes, el $12,5 \%$ ya estuvo alguna vez embarazada; de estas, el 9,9\% eran madres y el $2,6 \%$ estaban gestando por primera vez, y el $25 \%$ de estas chicas abandona el colegio por un embarazo. Los mayores porcentajes de adolescentes que son madres o que estaban embarazadas se presentan en las mujeres con educación primaria (33,9\%), en las residentes de la selva $(25,3 \%)$, entre las que se encuentran en el quintil inferior de riqueza $(22,4 \%)$ y en el área rural $(19,7 \%)$. Mientras tanto, los menores porcentajes se aprecian en Lima Metropolitana (8,3\%), entre aquellas con educación superior y en las del quintil superior de riqueza $(8,6 \%$ y $2,5 \%$, respectivamente) (Instituto Nacional de Estadística e Informática, 2012; El Comercio, 2011; De Irala y otros, 2011; Ministerio de Salud, 2012).

Por otro lado, la falta de información adecuada y de acceso a métodos anticonceptivos y las prácticas sexuales de riesgo, como tener múltiples parejas, conllevan a una alta incidencia y prevalencia de ITS, incluido el sida. En 2010, los casos nuevos notificados de VIH/Sida en jóvenes de 15 a 24 años de edad fue el 17,7. Si se agregan aquellos que son adultos, el porcentaje de las personas infectadas con el VIH que fueron contagiadas durante la adolescencia se incrementa (PNUD, s/f). 
Sobre el aborto, los datos indican que en Perú se reporta que una de cada diez mujeres que ingresan con una complicación de aborto ilegal es adolescente. Además, de las mujeres hospitalizadas por aborto en el Perú, $10,4 \%$ tienen de 15 a 19 años; en tanto, de las mujeres que abortan en el Perú, el 14\% tiene menos de 20 años. Asimismo, hace varios años se mostró que el aborto séptico, que es una forma indirecta de medir los abortos inducidos, tenía una tendencia a aumentar en las adolescentes a partir de 1990 en adelante. Acerca de la mortalidad materna en adolescentes, se encontró que $11 \%$ de las muertes maternas ocurre en mujeres de 10 a 19 años y que el aborto fue causa de más de $50 \%$ de esas muertes (Instituto Nacional de Estadística e Informática, 2012; Távara-Orozco, 2004).

Asimismo, los adolescentes se desenvuelven en un contexto sociocultural donde existen creencias y valores con respecto a su sexualidad, y donde la desinformación está asociada a la actividad sexual sin precauciones, ya que las fuentes de información y orientación en materia de educación sexual rara vez están a su alcance (ProJoven, 1999).

Según algunos autores (Portilla Valdivia y otros, 1997; Bendezú y otros, 1992; Salazar y otros, 1996), las pautas y normas de comportamiento sexuales se van formando mediante un proceso llamado socialización, donde intervienen la familia, la escuela, los medios de comunicación, los grupos de pares y la religión. Estos medios enseñan la forma en que se deben comportar socialmente los hombres y las mujeres con personas del mismo sexo y con el sexo opuesto. Pero, en muchos casos, estos medios brindan una información distorsionada, con lo cual confunden a las personas o hacen que acepten determinada información como verdadera. Asimismo, la sociedad transmite valores, costumbres y creencias que determinan los roles de género, generalmente en relación con su sexo biológico. Por lo tanto, las formas de ser hombre y de ser mujer se aprenden socialmente: la persona no nace con ellas.

Por ello, se reconoce a la familia como el núcleo básico donde «deberían» brindarse los primeros conocimientos sobre educación sexual, porque son los padres quienes, por estar más ligados afectivamente a sus hijos, podrían dar a este tema una dimensión positiva y saludable (Balarezo, 2005; Verau, s/f). Esta educación sexual debe empezar mediante el ejemplo de los padres, de tal manera que este ejemplo sea el reflejo de lo que los hijos imitarán inconscientemente a lo largo de sus vidas (Maestre, 2002).

En tal sentido, las actitudes sexuales son la forma como percibimos la sexualidad: positiva, negativa, neutra, responsable, irresponsable, valiosa, peligrosa, etc. Las actitudes se forman a partir de experiencias personales, modelos de personas importantes en nuestra vida y por aquello que se 
enseña a través del sistema educativo y los medios masivos. La primera actitud sexual que aprendemos viene del hogar. El niño nace entre brazos y es acariciado, tocado y marcado por las personas que lo aman. De esta experiencia de vida, las personas aprenden que el contacto físico no solo comunica afecto, sino que lo aumenta. También se aprende que el afecto y el contacto físico crecen cuando la relación es estable y permanente (Cruz Ruiz, s/f).

Otra actitud sexual importante que se aprende en casa es que existe diferencia entre el contacto conyugal (entre esposos), padres e hijos, de hermanos, parientes lejanos, de amigos y de conocidos. En la familia se modela cada uno de estos contactos, sin necesidad de enfatizarlos verbalmente. Los padres se abrazan, se besan, se acarician, se dicen frases, se acuestan juntos y expresan su sexualidad de formas distintas de las expresiones que tienen hacia otros miembros de la familia. Por otro lado, los padres a menudo caen en el dilema de si proporcionar o no educación sexual a los jóvenes; esto lo hacen sin darse cuenta, dado que lo están haciendo día a día a través de sus reacciones, actitudes y otras conductas cotidianas (Cruz Ruiz, s/f).

Sin embargo, la realidad todavía está lejos de hacer efectiva estas convicciones, porque en la mayoría de los casos los padres no están preparados para responder a preguntas sobre sexualidad o consideran este tema un tabú. Por eso, los adolescentes se quejan de que sus padres no están dispuestos a enseñarles aspectos de la sexualidad, porque los adultos se sienten incómodos al hablar sobre este tema (Balarezo, 2005; Cruz Ruiz, s/f).

Para evitar lo anterior, la educación sexual debe brindarse a temprana edad y según la edad del hijo, y en este sentido la primera responsabilidad la tienen los padres; sin embargo, muchos prefieren esperar a que los hijos entren a la pubertad para hablar sobre el tema. Una estrategia a utilizar es introducir estos temas en momentos cotidianos. Para ello, se pueden utilizar estímulos de cosas que suceden día a día en la televisión, las películas o los periódicos (Asociación Guatemalteca de Educación Sexual, 1990; El Comercio, 2013).

Pero en la realidad muchos padres han crecido donde la sexualidad no se trataba abiertamente y por dicha falta de conocimientos los hijos podrían descalificar a sus progenitores. Pero, en la mayoría de los casos, los padres tiene mitos, tabúes y temores al tratar el tema de la sexualidad con sus hijos, como: hablar de sexo fomenta la liberación sexual de los jóvenes; si se habla de sexo, se pierde el respeto de los hijos; hay que ser expertos para hablar sobre el tema; hablar de sexo es responsabilidad de la escuela; cuando el hijo tenga más edad, se puede hablar de ello; 
la orientación sexual es asunto de la madre; los padres deben hablar de sexualidad con los hijos y las madres, con las hijas; se tiene que ser amigo del hijo o la hija y eso le ocurre a los hijos de otras personas, no a mis hijos (Asociación Guatemalteca de Educación Sexual, 1990).

Además, el papel de los padres no es el de instructores, sino el de consejeros abiertos a escuchar las inquietudes de sus hijos; por ello, las respuestas de los padres deben aportar claridad y calma al hijo. Sea cual fuere el tema, se debe brindar información adecuada según la edad y características de los hijos. Asimismo, la forma como se aborde cada tema dependerá de los valores de cada familia (El Comercio [a], s/f; El Comercio [b], s/f). En tal sentido, la adolescencia puede ser una oportunidad de consolidar sus valores, fortalecerse mediante el diálogo y ofrecer al hijo las herramientas necesarias para ir convirtiéndose paulatinamente en un adulto (El Comercio (c), s/f).

Por ello, los adolescentes buscan la información que necesitan en sus pares (los que también están mal informados); se suman a ello los mensajes distorsionados (erotismo) que sobre sexualidad difunden los medios de comunicación, con lo cual se confunde más a los adolescentes en vez de ayudarlos a solucionar sus dudas e inquietudes (Balarezo, 2005; El Comercio [b], s/f; Chávez, Petrzelová y Zapata, 2009).

Lo recomendable es que se pueda hablar abiertamente con los padres de todos los temas: sexualidad, drogas, alcohol, etc. Será mejor recurrir a los padres en caso de que se tenga una duda: con su experiencia, ellos tienen una respuesta más acertada que la que pueden tener otros adolescentes. Desafortunadamente, no todos los padres piensan así. Algunos tienen temor a establecer este tipo de comunicación porque no saben qué responder ni cómo resolver las dudas con sus hijos (Pick de Weiss y Vargas-Trujillo, 1992).

Asimismo, se menciona que los jóvenes no carecen de información sobre la sexualidad, sino que disponen de un tipo de conocimiento «cotidiano» o «mitos» elaborados sobre nociones aportadas por pares, adultos próximos, medios de comunicación y otras personas, que no reúnen los requisitos del conocimiento científico, pero que les permite encontrar respuestas a dudas y preguntas. Además, distintas investigaciones muestran que el comportamiento sexual es modelado por distintas fuerzas sociales (estereotipos de género, reputación social, «confianza» en la pareja sexual, etc.) (Marston y King, 2006; Arditi y otros, 2004).

Asimismo, las investigaciones revelan que los padres no hablan abiertamente de sexualidad con sus hijos. La comunicación suele ser especialmente difícil durante la adolescencia, sobre todo si no se ha tenido 
costumbre de hacerlo en etapas anteriores. Además, en las charlas de las mujeres y los varones adolescentes con adultos, y a través de algunos silencios, se transmiten valores y normas que se ponen en juego en sus opiniones y comportamientos sexuales (Jones, 2010).

Los adultos suelen brindar orientaciones normativas sobre la actividad sexual que suele sintetizarse en lo siguiente: para las mujeres: «en lo posible no tengas relaciones sexuales durante tu adolescencia pero, en caso de tenerlas, que sea con un novio, por amor y utilizando algún método de prevención del embarazo y las infecciones de transmisión sexual»; para los varones: «puedes tener relaciones sexuales durante tu adolescencia siempre y cuando uses preservativos». Esto se debe a: 1) el reconocimiento de los adultos de que una proporción importante de adolescentes tiene relaciones sexuales, más allá de su valoración al respecto; 2) la extendida percepción del embarazo en la adolescencia como un problema que afecta al proyecto de vida de las mujeres; 3) la visibilidad de la epidemia del VIH/Sida a través de los medios de comunicación; y 4) el debate público sobre salud sexual y reproductiva (Jones, 2010).

En un estudio realizado en Argentina, se encontró que: 1) pareciera que es más fácil a las hijas recurrir a los padres; 2 ) un $48 \%$ de adolescentes no dialoga con sus padres; 3 ) las hijas prefieren comunicarse con la madre y los hijos con el padre; 4) dos tercios de los adolescentes dialogan con sus maestros sobre temas relacionados con el cuerpo y la sexualidad; 5) los adolescentes establecen más frecuentemente el diálogo con sus propios pares; y 6) los que hablan con sus padres tienen mayor predisposición de hablar con sus maestros (Pantelides, 1991).

En un estudio realizado en Lima (Perú) en colegios estatales nocturnos, se encontró que algunas alumnas manifestaban haber recibido información sobre sexualidad de sus profesores, madres y amigas, y que la mamá y la amiga eran las personas a quienes más recurren para dialogar sobre sus dudas. En los varones se informó que recurren como primera fuente a los amigos. Otro estudio realizado en Lima señala que el grupo de pares son los informantes más importantes en la formación de las opiniones de los adolescentes sobre la iniciación sexual; luego vienen los padres y, en última instancia, los profesores (Marston y King, 2006; Jones, 2010).

\section{Conclusiones}

La sexualidad es un componente psicosocial importante en el desarrollo de todo ser humano, porque sienta las bases para que las personas se relacionen entre sí, debido a que se producen un intercambio de conocimientos, actitudes, prácticas, valores, etc., que en el futuro pueden influir en las relaciones con el sexo opuesto y/o la pareja. 
Una etapa de la vida donde la sexualidad ejerce mucha influencia es la adolescencia, porque se incrementan las relaciones sociales y se inicia la atracción hacia el sexo opuesto; por lo tanto, los adolescentes se encuentran entre los valores aprendidos en casa y los que van aprendiendo con sus pares; dichos valores son importantes, porque, en muchos casos, son los que van a perdurar y guiar los actos de toda la vida.

Los adolescentes aprenden sobre la sexualidad mediante los medios de socialización como la familia, el colegio, los amigos, la religión y los medios de comunicación. Estos medios enseñan la forma en que se deben comportar socialmente los hombres y las mujeres con personas del mismo sexo y con el sexo opuesto. Pero, en muchos casos, estos medios brindan una información distorsionada, con lo cual confunden a los adolescentes o hacen que acepten determinada información como verdadera.

Los especialistas en el tema opinan que el primer lugar donde se aprenden cuestiones relacionadas con la sexualidad es el hogar, porque es ahí donde se observan las relaciones entre el padre y la madre, y de estos con los hijos. Además, también puede ser el lugar donde los hijos absuelven sus primeras dudas sexuales por la confianza que tienen con sus padres.

Lamentablemente, muchas veces los padres de familia no manejan la información adecuada, debido a que no han recibido ningún tipo de orientación o tienen ciertos prejuicios sobre el tema. En tales casos, los padres reaccionarán ante las preguntas de sus hijos evitando hablar sobre el tema o con respuestas distorsionadas, o reprimiéndolos o castigándolos porque piensan que sus hijos no están aún en edad para hablar sobre ello. Esto conlleva a que los adolescentes no tengan la suficiente confianza como para hablar de sexualidad con sus padres, por lo que recurren a los amigos (quienes se encuentran en la misma situación) o a revistas no especializadas en el tema, en las que se resalta la pornografía más que la sexualidad humana.

A ello se suma que, cuando se habla de sexualidad, además de la carga valorativa que significa, existen infinidad de mitos y tabúes que la rodean. Estas creencias son mencionadas y aceptadas socialmente y, en el peor de los casos, se transmiten de generación en generación, sin que exista un sustento científico para aceptar su veracidad. Estos mitos no son exclusivos de una clase social, dado que existen personas de todos los estratos socioeconómicos que nunca han recibido algún tipo de información sobre el tema. 


\section{REFERENCIAS BIBLIOGRÁFICAS}

Arditi, Z., Zorrilla, P., Lamy, P. y Pasqualini, D. (2004). Sexualidad adolescente: información y riesgo. Revista del Hospital de Niños Ricardo Gutiérrez, 46, pp. 203-208.

ASDE (2002). Desarrollando las habilidades sociales en la educación sexual de @@s adolescentes. Módulo de capacitación en salud sexual y reproductiva. Arequipa: Pathfinder, Prisma y Usaid.

Asociación Guatemalteca de Educación Sexual (1990). ¿Qué es la educación sexual? Guatemala: Asociación Guatemalteca de Educación Sexual.

Balarezo, G. (17 de enero 2005). ¿Por qué impartir educación sexual en los adolescentes? Gestión Médica, 23, 19.

Bendezú, A., Elna, A., Mejía, M. y Londoña, C. (1992). Desarrollo de la sexualidad humana. En Educación sexual para jóvenes y adultos (pp. 16-17). Lima: Asociación Perú-Mujer y Ministerio de Educación.

Chávez, M., Petrzelová, J. y Zapata, J. (2009). Actitudes respecto a la sexualidad en estudiantes universitarios. Enseñanza e Investigación en Psicología, 14, pp. 137-151.

Cruz Ruiz, L. A. (s/f). Educación sexual. En Representación social de la educación sexual de los padres de familia con hijos adolescentes (tesis). Universidad Autónoma Metropolitana, México D. F.

De Irala, J., Osorio, A., Carlos, S., Ruiz-Canela, M. y Lopez-del Burgo, C. (2011). Mean Age of First Sex: Do They Know What We Mean? Arch Sex Behav, 40, pp. 853-855.

El Comercio (a). (s/f). ¿De dónde vengo? Sexualidad infantil. En Guía para padres, fascículo 16, pp. 122-125.

(b). (s/f). La transición. Camino a la adultez. En guía para padres, fascículo 25, p. 196.

(c). (s/f). Citas, relaciones de pareja y sexo. Los primeros amores. En Guía para padres, fascículo 26, pp. 228-231.

(30 de octubre de 2011). Háblame de sexo (pp. 12-13).

Instituto Nacional de Estadística e Informática (2012). Encuesta demográfica y de salud 2011. Lima: INEI.

Jones, D. E. (2010). Diálogos entre padres y adolescentes sobre sexualidad: discursos morales y éticos en la reproducción de las desigualdades de género. Interface-Comunicação Saúde Educação, 14 (32), pp. 171-182.

López Reyes, L. y La Rosa, L. (1993). Capacitando profesionales de salud para la atención integral de adolescentes con énfasis en salud reproductiva. Lima: Proyecto Desarrollo de Servicios para Adolescentes Subregión II Lima Sur. 
Maestre, F. (2002). Educación sexual en los colegios. En Era tabú. Adolescentes. Guía de sexualidad para la familia (tomo 2) (p. 59). Lima: Grupo Santillana.

Marston, C. y King, E. (2006). Factors that Shape Young People's Sexual Behavior: a Systematic Review. Lancet, 368, pp. 1581-1586.

Ministerio de Educación (1998). Desarrollo psicosocial del adolescente. En Guía de educación familiar y sexual. Primer grado de Educación Secundaria (p. 25). Lima: Ministerio de Educación.

Ministerio de Salud (2012). Plan multisectorial para la prevención del embarazo en adolescentes 2012-2021 (pp. 7-9). Lima: Ministerio de Salud.

Pantelides, A. (1991). Fecundidad en la adolescencia en la Argentina. Buenos Aires: Centro de Estudios de Población.

Pick de Weiss, S. y Vargas-Trujillo, E. (1992). Introducción. En Yo, adolescente. Respuestas claras a mis grandes dudas (pp. 13-14). México D. F.: Editorial Planeta.

PNUD (s/f). ¿Cómo afrontar la problemática del VIH/SIDA a nivel nacional? Recuperado de http://www.undp.org/content/peru/es/home/ourwork/ hiv_aids/in_depth/

Portilla Valdivia, A., Soto Malca, G. y Leyton Muñoz, C. (1997). Sexo y sexualidad. En Educación sexual para adolescentes (pp. 16-18). Arequipa: Ceder y FNUAP.

PROJoven (1999). Módulo de sexualidad y salud sexual y reproductiva para jóvenes. Lima: Ministerio de Trabajo y Promoción del Empleo.

Rubarth, G. (1994). La adolescente embarazada. Buenos Aires: Grupo Editor Latinoamericano.

Salazar, V., Chirinos, J., Reátegui, L., Bardales, O., Alatrista, M. y Gallegos, D. (1996). Conociendo la sexualidad y los derechos sexuales. En Caminemos juntos hacia una sexualidad integral (pp. 23-24). Lima: IEPO y Universidad Peruana Cayetano Heredia.

Somos (2013). Hablemos de sexo. Año XXVI, 1378, pp. 88.

Soubies, M. E., González Velardo, M. J., Jorge, M. M. y otros (2010). Adolescentes de hoy... ¿mitos de ayer? Revista del Hospital de Niños de Buenos Aires, 52 (239), pp. 534-539.

Távara-Orozco, L. (2004). Contribución de las adolescentes a la muerte materna en el Perú. Ginecología y Obstetricia, 50 (2), pp. 111-122.

Verau, D. (1996). Los padres y la orientación sexual de la salud reproductiva. En Data-5. Sociedad, Salud y Reproducción (pp. 3-5). Lima: Prosar, Universidad Peruana Cayetano Heredia. 
(s/f). Comunicación familiar. En Hablemos de eso... (pp.

13-14) Lima: Educa.

Recibido: setiembre de 2015

Aceptado: diciembre de 2015 